\title{
Smart Objects Presence Facilitation in the Internet of Things
}

\author{
Ahmad Reza \\ Parnian $^{1}$
}

\author{
Mohammad Reza \\ Parsaei $^{1}$ \\ ${ }^{1}$ Department of Computer Engineering and IT, \\ Shiraz University of Technology, Shiraz, Iran \\ Reza Javidan ${ }^{1}$
}

Reza Mohammadi ${ }^{1}$ different techniques in error detection and flow control, IOT is supposed to present a reliable end-to-end communication too.

\begin{abstract}
Internet of Things (IOT) vision is to connect almost everything to the Internet. IOT includes various networks such as Low-power and Lossy Network (LLN). LLN is a network of small smart devices communicating through low power and low-bitrate medium. Enhanced computation power of LLN small devices is a step toward the IOT reality. Internet Engineering Task Force (IETF) thinks about connecting LLNs to current IP based Internet. Small smart LLN devices benefit from constrained resources such as memory and power. So IETF has designed a set of special protocols in order to facilitate LLN devices Internet connection. LLN special characteristic requires noticeable modifications of protocols used in MAC, Routing and Application layers too. In this paper IOT concept and visions are presented and a survey is done on standards and protocols devised in order to connect LLN to the Internet. An overview of the most prevalent IOT applications is presented too.
\end{abstract}

ABSTRACT

\section{Keywords}

IOT, 6LOWPAN, RPL, COAP.

\section{INTRODUCTION}

IOT as a new emerging evolution in networking has found a great place in researching area recently. IOT suggests enhanced connection of systems, devices and services beyond machine to machine communications. The recent advances in microcontroller, battery, microelectronic and low-power radio technologies gave the idea of connecting smart objects to the Internet. IOT provides the interconnection of all computation capable devices with the current Internet infrastructure and covers a variety of domains, protocols and applications. Smart phones, sensors, RFIDs, embedded devices, actuators and almost everything will be connected to the Internet. By 2025 everything in daily life will be a part of IOT, home Internet nodes will be home appliances, smart meters, lights, temperature and smoke sensors, Cameras, etc. [1]. IOT will have a significant impression on everyday life. The behavior of humanity is changed according to the implemented technologies in modern houses, for example a new technology assisting human being in remote monitoring and controlling. IOT impression on business is in industrial automation, logistic, power grid, intelligent transportation system, etc.

There are different definitions of IOT as it covers a wide domain of concepts. IOT is known as a network of uniquely addressed interconnected smart objects obeying standard communication protocols [2]. IOT is also services that are interfaced by smart identified things deployed in various spaces. These kinds of devices are capable to interact and communicate directly through the Internet [3]. Like the reliable communication that current Internet provides through
Radio Frequency Identifier (RFID) was the starting point of IOT concept. Scientists thought about smart and intelligent devices which are connected to the Internet. Devices are tiny sized with limited resources: low-power (battery), limited memory and computation power using low-rate radio communication.

Wireless Sensor Network (WSN) underlies IOT. A sensor network is organized of sensor nodes collecting data from environment and forwarding to sink node in a multi-hop manner [4]. WSN is used in application domains like agriculture, industry, health care, etc. The main challenges in WSN are the scarcity of power, reliability and scalability. WSN is a subset of LLN. LLN is comprised of small devices with limitation in computation power, memory, energy and radio communication. Various works are done through the IOT realization by both academy and industry unions. Recent applications in LLN are considered to show the better performance in attendance to IOT. It can be said that simple LLN require more standard to act as a part of IOT. A numerous of devices in IOT are battery operated so it is necessary to endure the minimum possible computation and communication. Thus a protocol stack to be used in this case must require the minimum energy power.

IP is an extensible, stable, fully tested and implemented standard protocol in network communication which current infrastructure of Internet is based on, so IETF decided to use it on IOT devices. IPv4 suffers from limited address space and cannot be employed in IOT since the number of devices to be addressed uniquely is extremely high. Contrarily IPv6 provides a universally wide unique address space, enough to identify all smart small objects with. But IP is a heavy protocol to be used on resource limited Smart devices, It is essential to design an IP compliant communication stack which is based on IP enabling devices to attend in IOT. They require a protocol stack that takes into account limited power, lossy radio and low capacity memories. So it is essential to remove overhead, extreme meta-data, non-optimized protocols and algorithms [5] as possible as it is. IETF organized groups of developers to present different layers of protocols suited for constrained devices [6].

IOT visions are presented in section 2. Section 3 describes IEE 802.15.4 and 6LOWPAN technologies as the lower layer standards. RPL routing protocol is presented in section 4 . Section 5 is about COAP. IOT applications are explained is section 6 and finally section 7 presents the conclusion. 


\section{IOT VISIONS}

IOT covers many concepts as researchers have presented various definitions and ideas on IOT. So it is a complex task to understand what really IOT means according to the vast area of idea and technology it covers. There are two key areas that define IOT: the Internet network and things. Researchers, scientists and business companies defined IOT according to their interests, needs and working fields. Variety of objects exists in IOT such as sensors and actuators, mobile phones, RFIDs and smart meters. Figure1 depicts the Internet of Things vision.

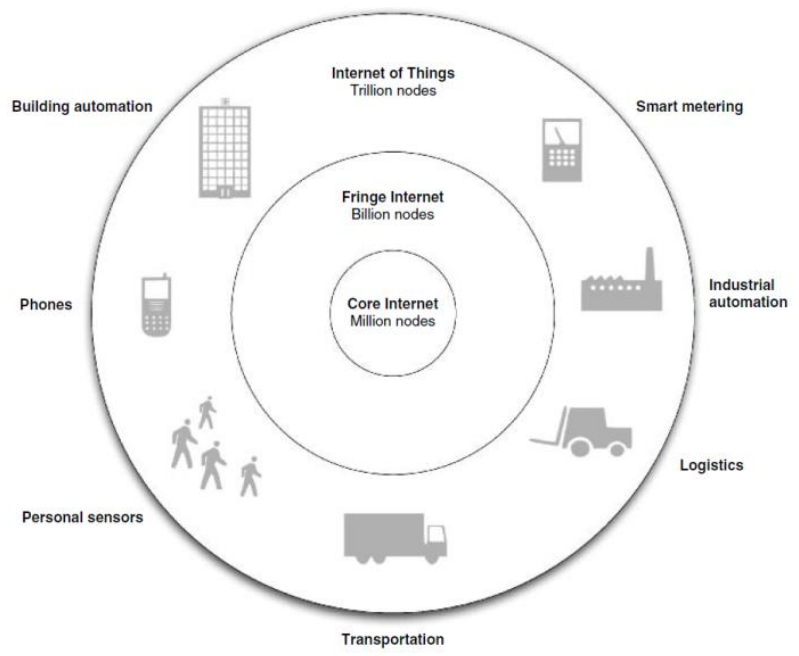

Fig 1: The Internet of Things Vision [6]

IOT is the intersection of three main visions: Internet oriented vision, Things oriented vision and Semantic oriented vision [3].

\subsection{Internet Oriented Vision}

Interconnecting smart objects is a challenging task in Internet oriented vision. The researchers focused on IP protocols as the solution. IP is the prevalent standard, and current infrastructure is based on IP. IP for Smart Objects (IPSO) [7] community formed in order to equip IP protocols for interconnecting smart objects. IPSO believes in IP capability in order to realize the IOT vision.

Objects are supposed to get compliant with IP protocols as they are the key protocols in the Internet. A Sensor object can reach to a level of computation power to be identifiable and monitored through unique IP address.

\subsection{Things Oriented Vision}

The vision came from simple things recognized by Radio Frequency Identification (RFID) tags. The idea was to organize a wide network of things which were identifiable through unique Electronic Code Product (EPC) stored on RFID tag. These standard provided awareness of object place and status. This was the starting point of IOT vision and IOT is not just a global EPC system with RFID objects. The idea was soon expanded to sensor networks. Sensors fulfill the concept of things. A Part of the IOT data is the data sensed by the sensors deployed in environments.

An IOT vision statement is a world of things communicating with each other and computers to serve humanity with various services. A vision of IOT is proposed by [7] assuming IOT as a universal infrastructure connecting physical and virtual objects in order to facilitate provision of services and applications.

\subsection{Semantic oriented Vision}

IOT includes a huge number of smart objects around the world. A unique addressing mechanism is necessarily required to provide a huge address space enabling all devices to be uniquely identified. Huge number of devices results in massive amount of generated data. The data maybe redundant and raw so must be processed and represented in meaningful and understandable format. Semantic technology provides mechanisms to process data in interoperable format. Storing and representation of the generated data are issues handled in semantic oriented vision [8].

A large number of devices will attend in IOT generating huge amount of data. Representation, mining [9-11], searching, storing and retrieving the generated data are serious challenges. Semantic technologies can be employed as a solution.

\section{LOWER LAYERS STANDARDS}

This section is about the lower layers standards in the IETF proposed protocol stack. PHY and MAC layers technologies are presented and an adaptation layer devised to be located between MAC layer and network layer is introduced.

\subsection{IEE 802.15.4}

IEEE 802.15 Personal Area Network (PAN) working group designed IEEE 802.15.4 radio communication standard covering the PHY and MAC layers rules. The standard was first published in the year 2003. IEEE 802.15.4 is the most famous standard among low-power wireless communication technologies. It is a low-power, low-data-rate technology covering almost small area spaces. Because of the specified characteristics of the IEEE 802.15.4 standard, it has gained a lot of attention as a radio communication standard to be used with embedded devices and small resource-constrained equipments. IOT benefits from this technology too, as it satisfies the special condition that IOT devices face. Various Vendors produce microcontroller motes with IEEE 802.15 based radios used in WSN applications. IEEE 802.15.4 supports maximum rate of $250 \mathrm{~Kb} / \mathrm{s}$ and maximum 127 bytes packet length. IEEE 802.15.4 is used with enhanced radio management technologies to reach the power consumption of transferring, receiving and idle time to the minimum possible value. The radio transceiver goes to the sleep mode when there is nothing to send or to receive.

IEEE 802.15.4 was not well established for multi-hop networks since there were problems such as power hungriness and single channel operation. So IEEE 802.15.4e working group was organized to provide a solution. IEEE 802.15.4e enhanced the reliability and reduced the duty cycle.

\subsection{Glowpan}

IOT devices are desired to benefit from IP, although there are challenges in appliance of IPv6 over IEEE 802.15.4 networks. IP minimum packet size is 1280 byte while the Maximum Transmission Unit (MTU) for IEEE802.15.4 is only 127 bytes so fragmentation and reassembly are necessary tasks to be done by the data link layer. Noticeable header overheads created by upper layers leaves almost no available payload size for application layer data and sending the 40 byte packet header of IPv6 over IEEE 802.15.4 link is completely bandwidth wasting. So it is essential to facilitate transmission of IP packets over IEEE 802.15.4 link layer. An adaptation layer is needed to tackle the specified challenges and provide 
a solution to send long IP packets over the link layer supporting 127 byte packet length. IETF IPv6 over Low power WPAN (6LoWPAN) working group got the responsibility to facilitate transmission of IP packets over IEEE 802.15.4 networks and designed 6LOWPAN adaptation layer above the link layer, which segments IPv6 packets into the supported frame size by the lower layer. 6LOWPAN performs header compression, routing overhead reduction and other necessary steps as an adaptation layer. LOWPAN_HCI is the standard for header compression and LOWPAN_IPHC is designed to reduce the IPv6 overhead. 6LOWPAN defines other standards in order to simplify neighbor discovery and facilitate mesh routing and other routing requirements. 6LOWPAN is the main step toward the IOT realization and is a base work for other research and improvements in IOT [6]. Figure 2 depicts 6LOWPAN protocol stack.

\section{LoWPAN Protocol Stack}

\begin{tabular}{|c|c|c|}
\hline Application & \multicolumn{2}{|c|}{ Application protocols } \\
\hline Transport & UDP & ICMP \\
\hline Network & \multicolumn{2}{|c|}{ IPv6 } \\
\hline & \multicolumn{2}{|c|}{ LoWPAN } \\
\hline Data Link & \multicolumn{2}{|c|}{ IEEE 802.15.4 MAC } \\
\hline Physical & \multicolumn{2}{|c|}{ IEEE 802.15.4 PHY } \\
\hline
\end{tabular}

Fig 2: 6LOWPAN protocol stack [6]

\subsection{LoWPAN Frame Format}

6LOWPAN is an adaptation layer between IPv6 network layer and IEEE 802.15.4 MAC layer performing fragmentation and link layer forwarding of IPv6 packets [12]. IPv6 header compression is done by omitting the redundant information inferable by upper layers [13].

6LOWPAN uses four kinds of prefixed headers ( 2 first bits) for encapsulated datagrams:

- NO 6LOWPAN Header is used to differentiate when there are non-6LOWPAN compliant nodes in the same network so the packet is discarded by the 6LOWPAN nodes.

- Dispatch Header is devised for IPv6 header compression or link layer multicast and broadcast.

- Mesh Addressing Header provides the transmission over IEEE 802.15.4.

- Fragmentation Header is for datagrams which require more than one frame to be encapsulated in.

\section{ROUTING}

There are many challenges with 6LOWPAN that needs to be addressed by routing protocol. Low-bitrate, low-power and lossy link, battery operated devices and the multi-hop topologies are the most serious limitation which the routing protocol deals with. A network with the specified conditions is called Low-power and Lossy Network (LLN). IETF organized Routing Over Low-power and Lossy network (ROLL) working group to specify a suitable routing protocol for this kind of network. ROLL did extensive experiments on existing routing protocols to see if they satisfy routing requirements of applications specified in [14-17]. The experiments were done on a set of routing protocols such as Open Shortest Path First (OSPF), Intermediate System to Intermediate (IS-IS) and Optimized Link State Routing (OLSR) over LLN applications. The conclusion was that none of them can completely suit LLN routing requirements [18], so ROLL designed Routing Protocol for Low-power and Lossy Networks (RPL) (RFC 6550) [19].

RPL is suitable for various link layers even lossy links of networks with constrained nodes acting as routers. Such networks exist in building, home, urban and industrial automation. RPL performs fast network construction, routing information distribution and quick adaption versus changes in network.

RPL is usually used in networks which nodes create multi-hop paths toward the set of aggregator devices. Each aggregator builds a Destination Oriented Directed Acyclic Graph (DODAG) of nodes. The collector node is the root of the DODAG organized by other nodes. DODAG is constructed according to metrics and constraints of nodes and links. There is an Objective Function (OF) numerating metrics and constraints of a path provided by a node. The gained value from OF is called Rank which declares the quality of a path toward the root through a node. Rank decreases uniformly through each path from leaf nodes toward the root node. OF is identified with the DODAGID which is defined by root node and is assigned to a DODAG that a root creates. DODAG version number holds the number of DODAG reconstruction time.

RPL supports point-to-point (P2P), point-to-multipoint (P2MP) and multipoint-to-point (MP2P) traffic types. MP2P is the most prevalent traffic type in LLN applications. In MP2P traffic flow sensor nodes generate data and forward it toward the gateway to get out of the network. This type of traffic is mainly seen in monitoring applications. P2MP traffic flow is transmitted from root node to the other DODAG members and this kind of traffic can be the actuation command resulted from MP2P traffic. P2P traffic flow provides member nodes communication.

RPL performs in two modes of operations: storing mode and non-storing mode. In non-storing mode nodes do not hold routing tables. They just forward the received packet from their child toward their parent and vice versa. In storing mode each node holds a routing table of the nodes reachable through itself. In non-storing mode a P2P traffic packet has to visit the root and then goes down to reach the destination. But in storing mode the packet goes up until reaches the first common ancestor. A DODAG can be both in storing and nonstoring mode too.

\subsection{DODAG Construction}

A DODAG can be constructed of multiple nodes and a single root node. If there is more than one root node, network is divided to multiple DODAGs, each created by a root node. These DODAGs can construct a single virtual DODAG when there is a virtual root as LLN root parent. A root node can organize multiple DODAGs of network nodes and this is done with RPL INSTANCEID. When there are more than one application, for each of them an $\mathrm{OF}$ can be defined, so different DODAGs are constructed based on application traffic requirements formulated by $\mathrm{OF}$.

RPL benefits ICMPV6 control messages to build the DODAG. Nodes propagate routing information by control messages. DODAG Information Object (DIO) contains OF, 
Rank, metrics, constraints and so on. At the start of DODAG construction process, root multicasts a DIO. The receiver nodes select root as their parent and calculate their rank based on the OF specified in the DIO. After that, they update the DIO information and multicast it. When all nodes selected their parents, a DODAG is constructed. If a node receives a DIO that is not the first received one, will compare the rank of the parent in the DIO with its current parent. The node will select the parent specified in DIO if it suggests a lower rank, if not will add it to the holding parent list.

\subsection{Downward Routes}

After DODAG construction, leaf nodes generate DAO packets. DAO contains information about node parent set. In non-storing mode, other nodes unicast DAOs to root node. After that the root received all the DAOs from other nodes, it can define paths to them by recursive extraction of parent sets. In storing mode, nodes transmit unicast DAOs to their parents informing them about the nodes reachable through themselves. Parent stores information in its routing table, generates a new DIO and sends it toward its parent.

Trickle timer controls redundancy and propagation of DIOs in a network. When trickle timer expires DIO is forwarded. Each node holds a trickle timer which upper bound is defined Imax and the lower bound is set to Imin. When a node receives a DIO exactly like the previous DIO, the DIO counter is increased by one and when the counter reaches the predefined threshold, the counter is reset and trickle timer is doubled. When an event like node fault occurs or Trickle timer reaches the Imax value, it is set to Imin [20-21].

\subsection{Objective Function}

Objective Function calculates node Rank based on some metrics and constraints. Node remaining energy, hop count, link Expected Transmission count (ETX), link latency and link reliability are some of the metrics and constraints specified in RFC 6551[22]. OF extracts metrics and constraints from the received DIO by a node. Then numeric value of the metrics/constrains for that node is calculated and added to the Rank provided by its parent. The resulted summation is the node Rank. Rank is the quality identifier of the best route that a node provides to root.

IETF has defined two following OFs:

- Objective Function 0: OF0 mechanism is analogous to hop count calculation. The node rank is computed as below:

$\mathrm{R}(\mathrm{N})=\mathrm{R}(\mathrm{P})+$ Rank_increase

Where $R(N)$ is the node rank and $R(P)$ is the parent rank. Rank_increase is calculated by:

Rank_increase $=\left(\mathrm{Rf}^{*} \mathrm{Sp}+\mathrm{Sr}\right) *$ MinHopRankIncrease [23]

Where $\mathrm{Sp}$ is computed based on the link properties, $\mathrm{Sr}$ defines the maximum augmentation to $\mathrm{Sp}$ and $\mathrm{Rf}$ is a coefficient to regulate link property impress on the rank_increase.

- The Minimum Rank with Hysteresis Objective Function (MRHOF)

MRHOF tries to select routes with minimum path cost according to the specified metric. Route with less cost is selected if the difference with current path is more than the value that hysteresis defines. Hysteresis tries to stabilize and prevent parent switching when there is not noticeable metric improvement. Current parent is replaced with new parent if new rank differs with current Rank more than the
PARENT_SWITCH_THRESHOLD. MRHOF works based on the additive metrics determined in DIO [24].

\section{APPLICATION LAYER}

Application layer is situated above the Transport layer protocol stack. Transport layer in wired networks provides reliable communication and performs error detection and flow control. TCP is a heavy-weight protocol stack with significant control overhead to be employed in LLN. In order to have reliability and low control overhead, UDP protocol is used as transport layer protocol. UDP does not guarantee packet delivery and supports retransmission control mechanism at application layer.

\subsection{Constrained Application Protocol}

Wired networks are not limited in energy, communication medium and other factors. The wired networks application level protocols are not optimized to work in limited conditions so the integration with LLN will face problems. New protocols and techniques are essential for interoperability of LLNs and current networks.

Most of applications use RESTful web services. According to limited resources, a full implementation of RESTful applications such as HTTP is not possible, so a new specific application protocol for LLN was required. IETF organized Constrained RESTful Environments (CoRE) working group to specify a light weight application protocol for LLN. CoRE thought about REST architecture in LLN networks and intended to implement a web protocol compliant with requirements of constrained environment. They tried to implement subset of optimized REST existing in HTTP. The implementation result was Constrained Application Protocol (CoAP) [25] which is a RESTful web transfer protocol specified for resource-constrained devices. COAP defines network objects as resources identified by URI. COAP is not a compression of HTTP but implements a subset of HTTP methods.

COAP operates over UDP and is based on two-layer architecture. The lower layer interacts with UDP while the upper layer deals with request and response transmissions.

Although COAP is different in some techniques with HTTP, it acts as HTTP for LLNs. COAP is a constrained web protocol with low header overhead and parsing complexity which supports optional resource discovery, asynchronous message exchanges, simple proxy and cashing capabilities. The protocol facilitates machine-to-machine communication which is prevalent in smart energy and building automation.

COAP provides features like Multicast support, built-in discovery and asynchronous message exchanges. It is easily translated to HTTP in order to integrate with the existing web. COAP and HTTP are mapped to each other in order to provide internetwork communication. COAP-HTTP mapping allows LLN clients to access HTTP server resources and HTTP-COAP mapping enables HTTP user clients to have access on LLN nodes. There is a HTTP-COAP proxy at the border of two networks mapping HTTP to COAP and vice versa.

COAP is organized of two sub layers: request/response and message layer.

Message layer controls message exchanges between endpoints. Request and responses obey the same message format. ID is used for messages to be identifiable in case of duplicates and reliability issues. 
Four types of messages exist: Confirmable, Non-Confirmable, Acknowledgement and Reset.

- Confirmable are messages requiring a response. The response can be piggybacked in acknowledgement.

- Non-confirmable messages are not replied and acknowledged.

- Acknowledgement messages approve confirmable messages delivery and sometimes piggyback a response.

- Reset message is forwarded when a confirmable message processing is impossible.

Request/response layer is used to exchange resource method requests and data of response or request. COAP messages contain request and response semantics. Response/request meta-data such as URI and payload data type may be included too. In order to corresponding response to a request, Token option can be used. Request contains resource identifier, payload, meta-data of request and the operation that should be done on the resource.

A Response message is recognizable through the Code field in COAP header; COAP Response Code determines result of the requested operation on the resource.

COAP uses four basic methods: GET, POST, PUT and DELETE. GET is used to get a representation of the requested resource through its URI. POST requests the process of the representation enclosed in the request. PUT requests the update or creation of the resource with the enclosed representation and DELETE requests for deletion of the resource with the ID specified in request URI [25].

Cashing mechanism tries to decrease the network bandwidth consumption and response time by reusing the prior response messages. Response messages are stored with expiration time and it is reused when a prior response exists that satisfies a request.

\section{APPLICATIONS}

IOT leads to numerous applications development. Some of them are the improved version of WSN applications and some are completely new and have already been implemented. Most of the application areas result in higher quality level of life. Industrial automation and environmental monitoring applications have found new opportunities and service domains with the help of IOT. In the following some application areas are introduced.

\subsection{Smart Grid Communication}

Smart grid is an improvement of current power grid system. Today electricity power demand is increasing more and more and conventional power grid is not able to satisfy customers as it performs weakly in automation and information feedback. It is difficult and complex to balance the generated amount of electricity power with the required amount so outage occurs or electricity is wasted due to over generation. Since storing equipments are rare and so expensive, the unneeded generated electricity is wasted. Spare generators are turned on in peak load time and almost all of them use fossil fuels and release $\mathrm{CO} 2$ in to the air. Smart grid communication handles the problem by collecting information from customers and all intermediate infrastructures. Smart grid provides real time monitoring and control, dynamic pricing and outage prevention [26].

\subsection{Building Automation}

Monitoring, control and management of wide area buildings is a complicated task. WSN technology is used in building management. Building automation employs various automation and actuator applications. Heating, ventilation and Air Conditioning (HVAC), security control, camera monitoring, lighting and fire detection are the main applications employed in building automation. It is estimated that building automation system decreases energy consumption about $30 \%$. Sensors and controllers usually run a web server in order to provide information through Internet. Building automation requires a reliable and scalable communication [27-28].

\subsection{Intelligent Transportation}

Almost all vehicles will be equipped with sensors and actuators soon. They join IOT as computing process powered devices. Roads attend in IOT too. Sensors and tags are embedded in roads which are constantly monitoring the situation and sending collected information toward the traffic control center and other vehicles in order to ensure traffic safety. Intelligent transportation helps routing and provides travelers with transportation information.

Automobiles, public transportation and even trains will be capable to process and exchange information gathered by embedded sensors in the area. Drivers and passengers are aware of roads status so they can navigate and travel safer. It is possible to avoid collision and control and monitor hazardous material transportation. Security provider organizations can observe roads traffic. Optimized navigation is possible assisting in fuel consumption saving [29].

Various IOT technologies integration leads to efficient transportation services. Vehicles can be identifiable and traceable during travel. They can cooperate to provide information about road status, maintaining the safe distance from each other. In cities, highway traffic normalization is dependent on the adequate real-time information of streets and highways congestion.

\subsection{Logistic}

RFID is a replacement of barcodes and is used efficiently in supply chain management. RFID technology is a part of IOT realization. RFID tags are attached to products capable to maintain information about the producer, expiration date and production date.

RFID and Near Field Communication (NFC) can realize real time tracking and monitoring of all the supply chain steps. Raw material preparation, production procedure, storing, transportation, distribution, selling and after-sale services, all can be Monitored. Since it is possible to get product information in a real-time manner, enterprises and business entities can react to sudden changes in customer requirements quickly [30].

Innovation in logistics allows process efficiency improvement and new features development.

\subsection{Medical and Health Care}

IOT helps in healthcare significantly. Many applications came to existence by IOT in healthcare domain. Medication and medical equipment control, medical information management, personal health management and telemedicine are IOT applications in e-health field.

It is possible to monitor and control all steps of medication and medical equipments provision consisting of production, 
shipment and delivery. Through the RFID tags attached to Medical products, counterfeit products are easily recognizable by querying the public database with the RFID tag. RFID tags attached to the medicines in production line can store all the information collected by around environmental monitoring. So a medicine product contains the information about the production and storing environment and other places through the delivery process. Determining the quality and health of the medicine is completely possible with the collected information.

Medical information management provides doctors and nurses with the patient's electronic health profile including all personal information of patient about medical treatment, experiments, consumed drugs, etc.[31]

Telemedicine is a service realized by integration of medical, computer and communication technologies. Telemedicine improved the quality and availability of medical help. Telemedicine technology resulted in Health care cost reduction, remote monitoring and consultation [32].

Health Management development purpose is to collect health status of people and find out if any sign of disease exists. Health care centers analyze the receiving data which results in early understanding of disease appearance leading to lower medical cost and better chance of treatment. This is done through collection of human health status by body sensors. The collected data is forwarded toward the related organizations by household communication devices.

\section{CONCLUSION}

IOT is the recent emergence technology trying to connect almost all devices to the Internet. A wide address space of IPv6 and the success of IP in current Internet infrastructure motivated researchers to benefit from IPv6 in IOT realization. Small resource-constrained devices organizing LLN networks attend in Internet too. Specific protocol stack is essential to connect LLNs to the Internet, so IETF organized various working groups in order to design resource-constrained device suited protocols in different layers. We introduced the IOT concept, applications and the protocol stack designed for LLN devices. 6LOWPAN is an adaptation layer facilitating IP packets transmission on IEEE 802.15.4 link layer. RPL distance-vector routing protocol performs routing in LLN and COAP is designed as the application layer protocol for LLN. Although the reviewed protocol stack performs acceptable and realizes IOT, more work needs to be done in order to satisfy scalability and efficiency requirements, and there are still many issues requiring further researches.

IOT is now a great research area in academic environment. Many researchers and commercial organization are extensively working on it. Since IOT contains a wide area of technologies, it provides too many different aspects to be researched and optimized. Advancements in smart object products and optimization of their protocol stack are instances of areas that require more and more research and innovation. Hardware experts intend to design and produce smaller smart objects with more computational and energy power. At the same time software experts try to optimize and miniaturize operating system size. Network experts work on smaller size protocol stack too which consumes less battery energy. Consequently there are still many aspects to be investigated in IOT.

\section{REFERENCES}

[1] U.S. National Intelligence Council (NIC), Global Trends 2025: A Transformed World, NIC, Available online: www.dni.gov/nic/NI-2025-project.html, November 2008.

[2] INFSO D.4 Networked Enterprise RFID INFSO G.2 Micro Nanosystems in Co-operation with the Working Group RFID of the ETP EPOSS, "Internet of Things in 2020, Roadmap for the Future, Version 1.1," European Commission, Information Society and Media, Tech. Rep., May 2008.

[3] L. Atzori, A. Iera, and G. Morabito, "The Internet of Things: A survey," Computer Networks, vol. 54, no. 15, pp. 2787-2805, October 2010.

[4] R. Taheri, M. R. Parsaei and R. Javidan. "A New Method for Optimizing Energy Consumption in Wireless Sensor Networks Using Enhanced LEACH Protocol," Journal of Engineering and Applied Sciences, vol. 100, no. 3, pp. 576-581, 2016.

[5] A. Nabaei, M. Hamian, M. R. Parsaei, R. Safdari, T. Samad-Soltani, H. Zarrabi, and A. Ghassemi. "Topologies and performance of intelligent algorithms: a comprehensive review," Artificial Intelligence Review, pp. 1-25, 2016.

[6] Z.shelby, c.bormann, 6LoWPAN: The Wireless Embedded Internet, wiley, 2009.

[7] A. Dunkels, J.P. Vasseur, IP for Smart Objects, Internet Protocol for Smart Objects (IPSO) Alliance, White Paper \#1, September 2008.

[8] I. Toma, E. Simperl, Graham Hench, A joint roadmap for semantic technologies and the internet of things, in: Proceedings of the Third STI Roadmapping Workshop, Crete, Greece, June 2009.

[9] M. R. Parsaei, S. M. Rostami and R. Javidan. "A Hybrid Data Mining Approach for Intrusion Detection on Imbalanced NSL-KDD Dataset," International Journal of Advanced Computer Science and Applications, vol. 7, no. 6, pp. 20-25, 2016.

[10] M. R. Parsaei, R. Taheri and R. Javidan. "Perusing The Effect of Discretization of Data on Accuracy of Predicting Naïve Bayes Algorithm," Journal of Current Research in Science, (1), pp. 457-462, 2016.

[11] M. R. Parsaei, M. Salehi. "E-mail spam detection based on part of speech tagging;" 2nd International Conference on Knowledge-Based Engineering and Innovation (KBEI), Proceeding of the IEEE, pp. 1010-1013, 2015.

[12] G. Montenegro, N. Kushalnagar, J. Hui, and D. Culler, Transmission of IPv6 Packets over IEEE 802.15.4 Networks, RFC 4944, Internet Engineering Task Force RFC 4944, September 2007.

[13] J. Hui and P. Thubert, Compression Format for IPv6 Datagrams over IEEE 802.15.4-Based Networks, RFC 6282, Internet Engineering Task Force RFC 6282, September 2011.

[14] J. Martocci, P. D. Mil, N. Riou and W. Vermeylen, "Building Automation Routing Requirements in LowPower and Lossy Networks", IETF RFC 5867, 2010.

[15] A. Brandt, J. Buron, G. Porcu, "Home Automation Routing Requirements in Low-Power and Lossy Networks", RFC 5826, 2010. 
[16] K. Pister, P. Thubert et al., "RFC 5673: Industrial Routing Requirements in Low-Power and Lossy Networks," RFC 5673, 2009.

[17] M. Dohler, T. Watteyne, T. Winter, and D. Barthel, "Routing Requirements for Urban Low-Power and Lossy Networks", IETF RFC 5548, 2009.

[18] E. Ancillotti, R. Bruno and M.Conti, "The Role of the RPL Routing Protocol for Smart Grid Communications", IEEE Communications Magazine, Volume:51, Issue: 1pp. 75 - 83, January 2013.

[19] T. Winter, P. Thubert, A. Brandt, T. Clausen, J. Hui, R. Kelsey, P. Levis, K. Pister, R. Struik and J. Vasseur, "RPL: IPv6 Routing Protocol for Low-Power and Lossy Networks". IETF RFC 6550, March, 2012.

[20] P. Levis, T. Clausen, J. Hui, O. Gnawali and J. Ko, "The Trickle Algorithm”, RFC 6206, 2011.

[21] M. R. Parsaei, S. H. Khalilian and R. Javidan. "A Comparative Study on Fault Tolerance Methods in IP Networks versus Software Defined Networks," International Academic Journal of Science and Technology, vol. 3, no. 4, pp. 146-154, 2016.

[22] J. Vasseur, M. Kim, K. Pister, N. Dejean, and D. Barthel, "Routing Metrics used for Path Calculation in Low Power and Lossy Networks", IETF RFC 6551, March 2012.

[23] J. P. Vasseur, M. Kim, K. Pister, N. Dejean, and D. Barthe, Routing Metrics Used for Path Calculation in Low-Power and Lossy Networks, RFC 6552, Internet Engineering Task Force RFC 6552, March 2012.

[24] O. Gnawali and P. Levis, The Minimum Rank with Hysteresis Objective Function, RFC 6719, Internet Engineering Task Force RFC 6719, September 2012.
[25] Z. Shelby, K. Hartke and C. Bormann, and B. Frank. "Constrained Application Protocol (CoAP)", RFC 7252, 2012.

[26] V. C. Güngör, D. Sahin, T. Kocak, S. Ergüt, C. Buccella, , C. Cecati and G. P. Hancke, "Smart Grid Technologies: Communication Technologies and Standards", IEEE Transactions on Industrial Informatics, pp. 529 - 539, Volume:7, Issue: 4 NOV, 2011.

[27] M.Jung, C. Reinisch and W. Kastner," Integrating Building Automation Systems and IPv6 in the Internet of Things', Sixth International Conference on Innovative Mobile and Internet Services in Ubiquitous Computing, pp. 683-688, 2012.

[28] M. R. Parsaei and A. R. Parnian, "IPv6 based routing in building automation network", second International Conference on Knowledge-Based Engineering and Innovation (KBEI), pp.1025-1031, 2015.

[29] L. Chunli, " Intelligent Transportation based on the Internet of Things", 2nd International Conference on Consumer Electronics, Communications and Networks (CECNet), pp. 360 - 362, april, 2012.

[30] Z.ma, x.shang, x. fu, f.luo, " the architecturenand key technologies of internet of things in logistics", International Conference on Cyberspace Technology, pp. $464-468,2013$

[31] J. P. Varkey, D. Pompili, and T. Walls, "Human Motion Recognition Using a Wireless Sensor-based Wearable System", Personal and Ubiq-uitous Computing, pp.1-14, Sep. 2011.

[32] G. Churcher, G. Bilchev, J. Foley, R. Gedge, and T. Mizutani. "Experiences applying sensor web enablement to a practical telecare application", 3rd International Symposium in Wireless Pervasive Computing, 2008. 